\title{
ON CERTAIN RELATIONS AMONG THE THETA CONSTANTS*
}

BY

\section{J. I. HUTCHINSON}

In the case of the theta functions of two variables $(p=2)$, the theta constants (i. e., the ten functions with zero arguments) are well known to be expressible in terms of any four $\left(2^{p}=2^{2}=4\right)$ of them, the most interesting relations being those in which the characteristics of the four form a GöPEL quadruple. Thus, in the formulæ given by KRAUSE in $\$ 11$ of his book, Die Transformation der hyperelliptischen Funktionen erster Ordnung, we have certain combinations of the constants rationally expressed in terms of $\vartheta_{5}, \vartheta_{0}, \vartheta_{12}, \vartheta_{34}$ (formulæ (1)), while each constant is expressed irrationally in terms of the same (formulæ (3)).

In the following pages the corresponding results are given for the case of the general theta functions $(p=p)$ : viz., formulæ (I), (II) below are a direct generalization of formulæ (1) of KRAUSE just referred to, and (IV) is a generalization of (3), while (III) is a new relation not existing for the case $p=2 . \dagger$

I use the definitions and notations of Frobenius (Das Additionstheorem der Thetafunctionen, Crelle's Journal, vol. 89, 1880) and start with the formula (loc. cit., p. 201):

$$
\begin{aligned}
& \sum_{a=0}^{r-1}\left(P_{a}\right) \vartheta\left[A_{a}\right](u+v)(u-v)(a+b)(a-b) \\
&=\sum_{a}\left(P_{a}\right) \vartheta\left[A_{a}\right](u+b)(u-b)(a+v)(a-v) .
\end{aligned}
$$

In this formula $A_{0}=A, A_{1}, \cdots, A_{r-1}$ are the $r=2^{p}$ characteristics of a GöPEL system, and $P_{a}=A A_{a}$.

In (1) replace $u$ and $b$ by $u+C$ and $b+C$, where $C$ denotes any system of half periods. The formula becomes :

$$
\begin{aligned}
& \sum\left(P_{a} C\right) \vartheta\left[A_{a} C\right](u+v)(u-v)(a+b)(a-b) \\
& =(C) \sum\left(P_{a} C\right) \vartheta\left[A_{a}\right](u+b)(u-b)(a+v)(a-v)
\end{aligned}
$$

* Presented to the Society December 28, 1899. Received for publication December 28, 1899. $\dagger$ Special examples of these relations for the case $p=3$ were communicatcd to me by $\mathrm{Mr}$. $\mathbf{H}$. W. KUHN and suggested the generalizations here made. 
Putting $u=v=a=b=0$ we have

$$
\sum\left(P_{a} C\right) \vartheta^{4}\left[A_{\alpha} C\right]=(C) \sum\left(P_{a} C\right) \vartheta^{4}\left[A_{a}\right]
$$

In (2) replace $u$ by $u+P_{\beta}$. We obtain

$$
\begin{aligned}
& \sum\left(P_{a} C\right)\left(\begin{array}{l}
P_{\beta} \\
A_{a} C
\end{array}\right) \vartheta\left[A_{\alpha} P_{\beta} C\right](u+v)(u-v) \vartheta\left[A_{a} C\right](a+b)(a-b) \\
& =(C) \sum\left(P_{a} C\right)\left(\begin{array}{l}
P_{\beta} \\
A_{a}
\end{array}\right) \vartheta\left[A_{a} P_{\beta}\right](u+b)(u-b) \vartheta\left[A_{a}\right](a+v)(a-v) .
\end{aligned}
$$

Making the arguments zero we have

$$
\begin{aligned}
& \sum\left(P_{a} C\right)\left(\begin{array}{l}
P_{\beta} \\
A_{\alpha} C
\end{array}\right) \vartheta^{2}\left[A_{\alpha} P_{\beta} C\right] \vartheta^{2}\left[A_{\alpha} C\right] \\
&=(C) \sum\left(P_{a} C\right)\left(\begin{array}{l}
P_{\beta} \\
A_{\alpha}
\end{array}\right) \vartheta^{2}\left[A_{\alpha} P_{\beta}\right] \vartheta^{2}\left[A_{a}\right]
\end{aligned}
$$

This formula can be simplified. Suppose the GöPEL system to be represented in the form

$$
A B_{i}, \quad A_{\beta} B_{i} \quad\left(i=0,1, \cdots, \frac{1}{2} r-1\right)
$$

where $B_{0}$ is the characteristic zero. Then (4) can be written :

$$
\begin{aligned}
{\left[1+\left(P_{\beta}, C\right)\right] \sum_{i}\left(B_{i} C\right)\left(\begin{array}{l}
P_{\beta} \\
A B_{i} C
\end{array}\right) \vartheta^{2}\left[A B_{i} C\right] \vartheta^{2}\left[A_{\beta} B_{i} C\right] } \\
=(C)\left[1+\left(P_{\beta}, C\right)\right] \sum_{i}\left(B_{i} C\right)\left(\begin{array}{l}
P_{\beta} \\
A B_{i}
\end{array}\right) \vartheta^{2}\left[A B_{i}\right] \vartheta^{2}\left[A_{\beta} B_{i}\right]
\end{aligned}
$$

In reducing to this form we notice that the $B_{i}$ are contained among the $P_{a}$ and hence $\left(P_{\beta}, B_{i}\right)=1$.

The formula so obtained vanishes identically if $\left(P_{\beta}, C\right)=-1$. If $\left(P_{\beta}, C\right)=1$ we have

$$
\begin{aligned}
& \sum\left(B_{i} C\right)\left(\begin{array}{l}
P_{\beta} \\
A B_{i} C
\end{array}\right) \vartheta^{2}\left[A B_{i} C\right] \vartheta^{2}\left[A_{\beta} B_{i} C\right] \\
&=(C) \sum\left(B_{i} C\right)\left(\begin{array}{l}
P_{\beta} \\
A B_{i}
\end{array}\right) \vartheta^{2}\left[A B_{i}\right] \vartheta^{2}\left[A_{\beta} B_{i}\right]
\end{aligned}
$$

It is to be observed in this connection that

and

$$
\begin{aligned}
& \left(A B_{i}\right)\left(A_{\beta} B_{i}\right)=(A)\left(A_{\beta}\right)\left(P_{\beta}, B_{i}\right)=(A)\left(A_{\beta}\right), \\
& \left(A B_{i} C\right)\left(A_{\beta} B_{i} C\right)=(A)\left(A_{\beta}\right)\left(P_{\beta}, C\right)=(A)\left(A_{\beta}\right) .
\end{aligned}
$$


Consequently if $A$ and $A_{\beta}$ are of opposite character (i. e., one odd and the other even), formula (II) vanishes identically.

Again, replacing $u, v, a, b$ by $u+\frac{1}{2} P_{\gamma}, v+\frac{1}{2} P_{\gamma}, a+\frac{1}{2} P_{\gamma}, b+\frac{1}{2} P_{\gamma}$ in formula (3), we have

$$
\begin{array}{r}
\sum\left(P_{a} C\right)\left(\begin{array}{c}
P_{\beta} P_{\gamma} \\
A_{a} C
\end{array}\right) \vartheta\left[A_{a} C\right](a-b) \vartheta\left[A_{a} P_{\beta} C\right](u-v) \vartheta\left[A_{a} P_{\gamma} C\right](a+b) \\
\cdot \vartheta\left[A_{a} P_{\beta} P_{\gamma} C\right](u+v) \\
=(C) \sum\left(P_{\alpha} C\right)\left(\begin{array}{c}
P_{\beta} P_{\gamma} \\
A_{a}
\end{array}\right) \vartheta\left[A_{a}\right](a-v) \vartheta\left[A_{a} P_{\beta}\right](u-b) \vartheta\left[A_{a} P_{\gamma}\right](a+v) \\
\cdot \vartheta\left[A_{\alpha} P_{\beta} P_{\gamma}\right](u+b)
\end{array}
$$

Making the arguments zero we have

$$
\begin{aligned}
& \sum\left(P_{\alpha} C\right)\left(\begin{array}{c}
P_{\beta} P_{\gamma} \\
A_{\alpha} C
\end{array}\right) \vartheta\left[A_{a} C\right] \vartheta\left[A_{a} P_{\beta} C\right] \vartheta\left[A_{\alpha} P_{\gamma} C\right] \vartheta\left[A_{\alpha} P_{\beta} P_{\gamma} C\right] \\
& =(C) \sum\left(P_{\alpha} C\right)\left(\begin{array}{c}
P_{\beta} P_{\gamma} \\
A_{\alpha}
\end{array}\right) \vartheta\left[A_{a}\right] \vartheta\left[A_{\alpha} P_{\beta}\right] \vartheta\left[A_{\alpha} P_{\gamma}\right] \vartheta\left[A_{\alpha} P_{\beta} P_{\gamma}\right]
\end{aligned}
$$

This formula can be simplified by representing the characteristics of the GöPEL system in the form :

$$
A D_{j}, \quad A_{\beta} D_{j}, \quad A_{\gamma} D_{j}, \quad A A_{\beta} A_{\gamma} D_{j} \quad\left(j=0,1, \cdots, \frac{1}{4} r-1\right) .
$$

Equation (5) becomes :

(b)

$$
\begin{array}{r}
{\left[1+\left(P_{\beta}, C\right)\right]\left[1+\left(P_{\gamma}, C\right)\right] \sum\left(D_{j} C\right)\left(\begin{array}{l}
D_{j} \\
A A_{\beta} A_{\gamma}
\end{array}\right) \vartheta\left[A D_{j} C\right]} \\
\cdot \vartheta\left[A_{\beta} D_{j} C\right] \vartheta\left[A_{\gamma} D_{j} C\right] \vartheta\left[A A_{\beta} A_{\gamma} D_{j} C\right] \\
=\left[1+\left(P_{\beta}, C\right)\right]\left[1+\left(P_{\gamma}, C\right)\right](C)\left(\begin{array}{c}
C \\
A
\end{array}\right)\left(\begin{array}{c}
A_{\beta} A_{\gamma} \\
C
\end{array}\right) \sum\left(D_{j} C\right)\left(\begin{array}{l}
D_{j} \\
A A_{\beta} A_{\gamma}
\end{array}\right) \\
\cdot \vartheta\left[A D_{j}\right] \vartheta\left[A_{\beta} D_{j}\right] \vartheta\left[A_{\gamma} D_{j}\right] \vartheta\left[A A_{\beta} A_{\gamma} D_{j}\right] .
\end{array}
$$

If either $\left(P_{\beta}, C\right)$ or $\left(P_{\gamma}, C\right)$ is equal to -1 , formula (6) vanishes identically. If $\left(P_{\beta}, C\right)=\left(P_{\gamma}, C\right)=1$ we have

$$
\begin{array}{r}
\sum_{j}\left(D_{j} C\right)\left(\begin{array}{l}
D_{j} \\
A A_{\beta} A_{\gamma}
\end{array}\right) \vartheta\left[A D_{j} C\right] \vartheta\left[A_{\beta} D_{j} C\right] \vartheta\left[A_{\gamma} D_{j} C\right] \vartheta\left[A A_{\beta} A_{\gamma} D_{j} C\right] \\
=(C)\left(\begin{array}{l}
C \\
A
\end{array}\right)\left(\begin{array}{c}
A_{\beta} A_{\gamma} \\
C
\end{array}\right) \sum_{j}\left(D_{j} C\right)\left(\begin{array}{l}
D_{j} \\
A A_{\beta} A_{\gamma}
\end{array}\right) \vartheta\left[A D_{j}\right] \vartheta\left[A_{\beta} D_{j}\right] \vartheta\left[A_{\gamma} D_{j}\right] \\
\cdot \vartheta\left[A A_{\beta} A_{\gamma} D_{j}\right] .
\end{array}
$$


Further results are most easily obtained by starting with the formula :

$$
\begin{aligned}
& {\left[\sum_{a} \sqrt{P_{a}} \vartheta\left[A_{a}\right](u+v)(u-v)\right]\left[\sum_{a} \sqrt{P_{a}} \vartheta\left[A_{a}\right](a+b)(a-b)\right]} \\
& \quad=\left[\sum_{a} \sqrt{ } \overline{P_{a}} \vartheta\left[A_{a}\right](u+b)(u-b)\right]\left[\sum \sqrt{P_{a}} \vartheta\left[A_{a}\right](a+v)(a-v)\right],
\end{aligned}
$$

which is given in the memoir cited above (p. 200). We assume here that the characteristics $A_{a}$ are all even. Replace $u, b$ by $u+C, b+C$. We obtain :

$$
\begin{aligned}
& {\left[\sum\left(\begin{array}{c}
C \\
A_{a}
\end{array}\right) \sqrt{P_{a}} \vartheta\left[A_{a} C\right](u+v)(u-v)\right]\left[\sum\left(\begin{array}{c}
C \\
A_{a}
\end{array}\right) \sqrt{P_{a}} \vartheta\left[A_{a} C\right](b+a)(b-a)\right]} \\
& =\left[\sum\left(A_{a}, C\right) \sqrt{P_{a}} \vartheta\left[A_{a}\right](u+b)(u-b)\right]\left[\sum \sqrt{P_{a}} \vartheta\left[A_{a}\right](a+v)(a-v)\right] .
\end{aligned}
$$

Making the arguments zero and extracting the root we have

$$
\sum\left(\begin{array}{c}
C \\
A_{a}
\end{array}\right) \sqrt{P_{a}} \vartheta^{2}\left[A_{a} C\right]=\sqrt{\sum\left(A_{a}, C\right) \sqrt{P_{a}} \vartheta^{2}\left[A_{a}\right] \cdot \sum \sqrt{P_{a}} \vartheta^{2}\left[A_{a}\right]} .
$$

Multiply both members by $\sqrt{P_{\beta}}$, and add all the $2^{p}$ equations obtained by giving all possible combinations of signs to the radicals $\sqrt{P_{a}}, \sqrt{P_{\beta}}$. Using $\mathrm{S}$ to denote this summation and observing that $\mathbf{S} \sqrt{P_{a}} \sqrt{P_{\beta}}=2^{p}\left(P_{a}\right)$, or 0 according as $a$ is or is not equal to $\beta$, we have

(IV) $2^{p}\left(\begin{array}{c}C \\ A_{a}\end{array}\right)\left(P_{a}\right) \vartheta^{2}\left[A_{a} C\right]=\mathrm{S} \sqrt{P_{a}} \sqrt{\sum\left(A_{a}, C\right) \sqrt{P_{a} \vartheta^{2}\left[A_{a}\right] \cdot \sum \sqrt{P_{a}} \vartheta^{2}\left[A_{a}\right]}}$

This formula vanishes identically if $\left(A_{\alpha} C\right)=-1$.

The relation (IV) enables one to express all of the $2^{p-1}\left(2^{p}+1\right)$ theta constants homogeneously and algebraically in terms of $2^{p}$ of them, viz., the $\vartheta\left[A_{a}\right]$. Since the thetas depend on $\frac{1}{2} p(p+1)+1$ parameters (including the common multiplicative constant) it is evident that for $p>2$ formula (IV) gives a parametric representation of the theta constants with $2^{p}-\frac{1}{2} p(p+1)-1$ superfluous parameters; or, in other words, the $2^{p}$ parameters $\vartheta\left[A_{a}\right]$ satisfy this number of relations. These relations would seem to follow at once from formula (3), p. 204 of the memoir of Frobenius already cited when the arguments $u$ are made to vanish.

Cornele University, December, 1899. 